\title{
On the Singularly Perturbed Matrix Differential Riccati Equation
}

\author{
Zoran Gajic, Sarah Koskie, and Cyril Coumarbatch
}

\begin{abstract}
In this paper, the finite-time optimal control problem for time-invariant linear singularly perturbed systems is considered. The reduced-order pure-slow and pure-fast matrix differential Riccati equations are obtained by decoupling the singularly perturbed differential matrix Riccati equation of dimension $n_{1}+n_{2}$ into the regular differential matrix Riccati equation pure-slow of dimension $n_{1}$ and the stiff differential matrix Riccati equation pure-fast of dimension $n_{2}$. A formula is derived that produces the solution of the original singularly perturbed matrix Riccati differential equation in terms of solutions of the pure-slow and pure-fast reduced-order differential matrix Riccati equations and solutions of two reduced-order initial value problems. In addition to its theoretical importance, the main result of this paper can also be used to implement optimal filtering and control schemes for singularly perturbed linear systems independently in pure-slow and pure-fast time scales. An example for a catalytic fluid reactor model has been include to demonstrate the utility of the method.
\end{abstract}

\section{INTRODUCTION}

Singularly perturbed differential equations have been studied in mathematics [1], [2] and control systems engineering [3], [4], [5], and [6] for quite some time. These differential equations are characterized by coexistence of slow and fast variables with a small positive singular perturbation parameter $\varepsilon$ multiplying derivatives of the fast variables. Simultaneous presence of slow and fast phenomena causes numerical ill-conditioning [7] so an important goal in studying singularly perturbed systems of differential equations is to separate slow and fast variables. Since the initial work of Tikhonov [8], the singularly perturbed differential equations have been studied analytically by using different series expansion techniques and then matching corresponding terms [9], [1], and [2] to obtain the approximate solutions with accuracy of $O\left(\varepsilon^{k}\right), \quad k=1,2,3, \ldots$ for many important problems.

In 1972 a powerful transformation that produces the exact decomposition of linear systems of singularly perturbed differential equations into pure-slow and pure-fast (stiff) subsystems was obtained by Chang [10]. As a matter of fact, in [10], Chang studies a general boundary value problem of singularly perturbed linear systems. Since that time the Chang transformation has been used very often in the engineering literature of singularly perturbed linear control

Z. Gajic is with the Department of Electrical and Computer Engineering, Rutgers University, Piscataway, NJ 08854, USA gajiclece.rutgers.edu

S. Koskie is with the Department of Electrical and Computer Engineering, Purdue School of Engineering and Technology, IUPUI, Indianapolis, IN 46202, USA skoskiediupui.edu

C. Coumarbatch is a graduate student in the Department of Mathematics, Rutgers University, Piscataway, NJ 08854, USA cec@math.rutgers.edu systems. A new version of the Chang transformation is developed in [11]. A comprehensive overview of decoupling transformations for linear dynamical systems with emphasis on the work of Chang [10] can be found in [12]; see also [5], Chapter 3.

The decoupling transformation of Chang is limited to linear singularly perturbed initial and boundary value problems. The main goal of this paper is to introduce a transformation technique that produces the pure-slow pure-fast decomposition of the nonlinear singularly perturbed differential Riccati equation. In addition, we show how to obtain the solution of the original singularly perturbed differential Riccati equation in terms of solutions of the pure-slow and pure-fast reducedorder differential Riccati equations and solutions of two initial value problems.

Several authors have considered infinite horizon linear quadratic optimal control problems, including [13], and [14] and [15]. In this paper we will consider instead the finite horizon problem.

The paper is organized as follows. In Section II we introduce the singularly perturbed differential Riccati equation appearing in the context of the linear-quadratic optimal regulator problem [16] and [1]. Section III presents the transformation technique for pure-slow/pure-fast decomposition of the corresponding Riccati equation.

\section{SINGULARLY PERTURBED MATRIX DIFFERENTIAL RICCATI EQUATION}

Consider the singularly perturbed time-invariant linear system [16], [1], [2], and [3]

$$
\begin{aligned}
\dot{x}(t, \varepsilon)= & A_{1} x(t, \varepsilon)+A_{2} z(t, \varepsilon)+B_{1} u(t, \varepsilon), \\
& x(0, \varepsilon)=x_{0} \\
\varepsilon \dot{z}(t, \varepsilon)= & A_{3} x(t, \varepsilon)+A_{4} z(t, \varepsilon)+B_{2} u(t, \varepsilon), \\
& z(0, \varepsilon)=z_{0}
\end{aligned}
$$

where $x(t, \varepsilon)$ and $z(t, \varepsilon)$ are $n_{1}$ and $n_{2}$-dimensional slow and fast state vectors respectively, $u(t, \varepsilon)$ is an $m-$ dimensional vector control input, and $\varepsilon$ is a small positive parameter. Matrices $A_{i}, i \in\{1,2,3,4\}$, and $B_{j}, j \in\{1,2\}$, are constant and of appropriate dimensions. It is commonly assumed in the literature on singular perturbations that the fast subsystem matrix $A_{4}$ is nonsingular (standard linear singularly perturbed systems). A singular matrix $A_{4}$ defines nonstandard singularly perturbed linear systems [17]. With 
(1) consider the performance criterion

$$
\begin{gathered}
J=\frac{1}{2} \int_{0}^{t_{f}}\left\{\left[\begin{array}{l}
x(t, \varepsilon) \\
z(t, \varepsilon)
\end{array}\right]^{T} Q\left[\begin{array}{l}
x(t, \varepsilon) \\
z(t, \varepsilon)
\end{array}\right]+u^{T}(t, \varepsilon) R u(t, \varepsilon)\right\} d t+ \\
\frac{1}{2}\left[\begin{array}{l}
x\left(t_{f}, \varepsilon\right) \\
z\left(t_{f}, \varepsilon\right)
\end{array}\right]^{T} F(\varepsilon)\left[\begin{array}{l}
x\left(t_{f}, \varepsilon\right) \\
z\left(t_{f}, \varepsilon\right)
\end{array}\right]
\end{gathered}
$$

to be minimized along trajectories of (1). The following assumption is commonly used for the criterion penalty matrices.

Assumption 1: The penalty matrices in the performance criterion (2) are symmetric. In addition, the matrix $R$ is positive definite, and the matrices $Q$ and $F(\varepsilon)$ are positive semidefinite.

The well-known solution to the optimal control problem defined by (1) and (2) is given by

$$
u_{\text {opt }}(t, \varepsilon)=-\left(G_{1}(t, \varepsilon) x(t, \varepsilon)+G_{2}(t, \varepsilon) z(t, \varepsilon)\right)
$$

with

$$
G_{1}(t, \varepsilon)=R^{-1}\left(B_{1}^{T} P_{1}(t, \varepsilon)+B_{2}^{T} P_{2}^{T}(t, \varepsilon)\right),
$$

and

$$
G_{2}(t, \varepsilon)=R^{-1}\left(\varepsilon B_{1}^{T} P_{2}(t, \varepsilon)+B_{2}^{T} P_{3}(t, \varepsilon)\right)
$$

where the $P_{i}(t, \varepsilon), i \in\{1,2,3\}$, are the corresponding partitions of the positive semidefinite solution of the regulator matrix Riccati differential equation [16] and [1]

$$
\begin{aligned}
-\dot{P}(t, \varepsilon)= & A^{T}(\varepsilon) P(t, \varepsilon)+P(t, \varepsilon) A(\varepsilon) \\
& -P(t, \varepsilon) S(\varepsilon) P(t, \varepsilon)+Q, \\
& P\left(t_{f}, \varepsilon\right)=F(\varepsilon)
\end{aligned}
$$

with

$$
\begin{gathered}
A(\varepsilon)=\left[\begin{array}{cc}
A_{1} & A_{2} \\
\frac{1}{\varepsilon} A_{3} & \frac{1}{\varepsilon} A_{4}
\end{array}\right], \quad B(\varepsilon)=\left[\begin{array}{c}
B_{1} \\
\frac{1}{\varepsilon} B_{2}
\end{array}\right], \\
S(\varepsilon)=B(\varepsilon) R^{-1} B^{T}(\varepsilon), \\
Q=\left[\begin{array}{cc}
Q_{1} & Q_{2} \\
Q_{2}^{T} & Q_{3}
\end{array}\right] \quad \text { and } \quad F(\varepsilon)=\left[\begin{array}{cc}
F_{1} & \varepsilon F_{2} \\
\varepsilon F_{2}^{T} & \varepsilon F_{3}
\end{array}\right] .
\end{gathered}
$$

Note that the facts that the problem matrices are constant and Assumption 1 imposed on the penalty matrices, that is, $R=R^{T}>0, Q=Q^{T} \geq 0, F(\varepsilon)=F(\varepsilon)^{T} \geq 0$, guarantee the existence of the unique positive semidefinite symmetric matrix $P(t, \varepsilon)$ for all $t \in\left[0, t_{f}\right]$, see for example, [18].

The required solution of $(6), P(t, \varepsilon)$, is scaled to be compatible with the structure of the problem matrices as follows [16], [1]

$$
P(t, \varepsilon)=\left[\begin{array}{cc}
P_{1}(t, \varepsilon) & \varepsilon P_{2}(t, \varepsilon) \\
\varepsilon P_{2}^{T}(t, \varepsilon) & \varepsilon P_{3}(t, \varepsilon)
\end{array}\right]
$$

The partitioned form of (6) produces the following system of singularly perturbed matrix differential equations

$$
\begin{aligned}
-\dot{P}_{1}(t, \varepsilon)= & P_{1}(t, \varepsilon) A_{1}+A_{1}^{T} P_{1}(t, \varepsilon) \\
& +P_{2}(t, \varepsilon) A_{3}+A_{3}^{T} P_{2}^{T}(t, \varepsilon) \\
& -P_{1}(t, \varepsilon) S_{1} P_{1}(t, \varepsilon) \\
& -P_{1}(t, \varepsilon) S_{12} P_{2}^{T}(t, \varepsilon) \\
& -P_{2}(t, \varepsilon) S_{12}^{T} P_{1}(t, \varepsilon) \\
& -P_{2}(t, \varepsilon) S_{2} P_{2}^{T}(t, \varepsilon)+Q_{1}, \\
& P_{1}\left(t_{f}, \varepsilon\right)=F_{1} \\
-\varepsilon \dot{P}_{2}(t, \varepsilon)= & P_{1}(t, \varepsilon) A_{2}+P_{2}(t, \varepsilon) A_{4} \\
& +\varepsilon A_{1}^{T} P_{2}(t, \varepsilon)+A_{3}^{T} P_{3}(t, \varepsilon) \\
& -\varepsilon P_{1}(t, \varepsilon) S_{1} P_{2}(t, \varepsilon) \\
& -P_{1}(t, \varepsilon) S_{12} P_{3}(t, \varepsilon) \\
& -\varepsilon P_{2}(t, \varepsilon) S_{12}^{T} P_{2}(t, \varepsilon) \\
& -P_{2}(t, \varepsilon) S_{2} P_{3}(t, \varepsilon)+Q_{2}, \\
& P_{2}\left(t_{f}, \varepsilon\right)=F_{2} \\
& P_{3}(t, \varepsilon) A_{4}+A_{4}^{T} P_{3}(t, \varepsilon) \\
& +\varepsilon P_{2}^{T}(t, \varepsilon) A_{2}+\varepsilon A_{2}^{T} P_{2}(t, \varepsilon) \\
& -\varepsilon^{2} P_{2}^{T}(t, \varepsilon) S_{1} P_{2}(t, \varepsilon) \\
& -\varepsilon P_{2}^{T}(t, \varepsilon) S_{12} P_{3}(t, \varepsilon) \\
& -\varepsilon P_{3}(t, \varepsilon) S_{12}^{T} P_{2}(t, \varepsilon) \\
& -P_{3}(t, \varepsilon) S_{2} P_{3}(t, \varepsilon)+Q_{3}, \\
& P_{3}\left(t_{f}, \varepsilon\right)=F_{3} \\
-\varepsilon \dot{P}_{3}(t, \varepsilon)= &
\end{aligned}
$$

with

$$
S_{i}=B_{i} R^{-1} B_{i}^{T}, \quad i \in\{1,2\}, \quad \text { and } \quad S_{12}=B_{1} R^{-1} B_{2}^{T}
$$

The singularly perturbed system of matrix differential equations (9)-(11) has been studied analytically in [16] and [1] using asymptotic series expansions, and numerically in [19] by employing block diagonalization of the corresponding Hamiltonian matrix. Yackel and Kokotovic [20] obtain their results using control oriented assumptions (boundary layer controllability and boundary layer observability assumptions). These assumptions are relaxed in [1] into a set of linear algebraic requirements. One of the assumptions used in [1] will be needed for the purpose of this paper. It can be stated as follows.

Assumption 2: The fast subsystem Hamiltonian matrix is nonsingular, that is

$$
\operatorname{det}\left[\begin{array}{cc}
A_{4} & -S_{2} \\
-Q_{3} & -A_{4}^{T}
\end{array}\right] \neq 0 .
$$

In this paper we develop a new approach to solving (9)(11) by introducing a transformation technique that will allow us to decompose (9)-(11) into the reduced-order pureslow and pure-fast (stiff) differential Riccati equations. That will be achieved by extending the ideas from [19] and [21], where a nonsingular transformation has been used to block diagonalize the Hamiltonian system. In that direction, we will 
be able to obtain the solution of the original Riccati equation (6) in terms of solutions of the reduced-order pure-slow and pure-fast differential Riccati equations and solutions of two linear initial value problems.

\section{MAIN RESULT}

The Hamiltonian form of the optimal control problem defined in (1) and (2) is given by

$\left[\begin{array}{c}\dot{x}(t, \varepsilon) \\ \dot{z}(t, \varepsilon) \\ \dot{p}(t, \varepsilon) \\ \dot{q}(t, \varepsilon)\end{array}\right]=\left[\begin{array}{cccc}A_{1} & A_{2} & -S_{1} & -\frac{1}{\varepsilon} S_{12} \\ \frac{1}{\varepsilon} A_{3} & \frac{1}{\varepsilon} A_{4} & -\frac{1}{\varepsilon} S_{12}^{T} & -\frac{1}{\varepsilon^{2}} S_{2} \\ -Q_{1} & -Q_{2} & -A_{1}^{T} & -\frac{1}{\varepsilon} A_{3}^{T} \\ -Q_{2}^{T} & -Q_{3} & -A_{2}^{T} & -\frac{1}{\varepsilon} A_{4}^{T}\end{array}\right]\left[\begin{array}{c}x(t, \varepsilon) \\ z(t, \varepsilon) \\ p(t, \varepsilon) \\ q(t, \varepsilon)\end{array}\right]$

with costates $p(t, \varepsilon)$ and $q(t, \varepsilon)$ and boundary conditions

$$
\left[\begin{array}{c}
x(0, \varepsilon) \\
z(0, \varepsilon) \\
p\left(t_{f}, \varepsilon\right) \\
q\left(t_{f}, \varepsilon\right)
\end{array}\right]=\left[\begin{array}{c}
x_{0} \\
z_{0} \\
F_{1} x\left(t_{f}, \varepsilon\right)+\varepsilon F_{2} z\left(t_{f}, \varepsilon\right) \\
\varepsilon F_{2}^{T} x\left(t_{f}, \varepsilon\right)+\varepsilon F_{3} z\left(t_{f}, \varepsilon\right)
\end{array}\right] .
$$

Introducing the change of variables $q(t, \varepsilon)=\varepsilon r(t, \varepsilon)$ and interchanging the equations for $z(t, \varepsilon)$ and $p(t, \varepsilon)$, we get the standard linear singularly perturbed system

$$
\left[\begin{array}{c}
\dot{x}(t, \varepsilon) \\
\dot{p}(t, \varepsilon) \\
\varepsilon \dot{z}(t, \varepsilon) \\
\varepsilon \dot{r}(t, \varepsilon)
\end{array}\right]=\left[\begin{array}{ll}
\mathbf{A}_{\mathbf{1}} & \mathbf{A}_{\mathbf{2}} \\
\mathbf{A}_{\mathbf{3}} & \mathbf{A}_{\mathbf{4}}
\end{array}\right]\left[\begin{array}{c}
x(t, \varepsilon) \\
p(t, \varepsilon) \\
z(t, \varepsilon) \\
r(t, \varepsilon)
\end{array}\right]
$$

where

$$
\begin{array}{cc}
\mathbf{A}_{\mathbf{1}}=\left[\begin{array}{cc}
A_{1} & -S_{1} \\
-Q_{1} & -A_{1}^{T}
\end{array}\right], & \mathbf{A}_{\mathbf{2}}=\left[\begin{array}{cc}
A_{2} & -S_{12} \\
-Q_{2} & -A_{3}^{T}
\end{array}\right], \\
\mathbf{A}_{\mathbf{3}}=\left[\begin{array}{cc}
A_{3} & -S_{12}^{T} \\
-Q_{2}^{T} & -A_{2}^{T}
\end{array}\right], & \mathbf{A}_{\mathbf{4}}=\left[\begin{array}{cc}
A_{4} & -S_{2} \\
-Q_{3} & -A_{4}^{T}
\end{array}\right] .
\end{array}
$$

The boundary conditions for (16) are

$$
V\left[\begin{array}{c}
x(0, \varepsilon) \\
p(0, \varepsilon) \\
z(0, \varepsilon) \\
r(0, \varepsilon)
\end{array}\right]+N(\varepsilon)\left[\begin{array}{c}
x\left(t_{f}, \varepsilon\right) \\
p\left(t_{f}, \varepsilon\right) \\
z\left(t_{f}, \varepsilon\right) \\
r\left(t_{f}, \varepsilon\right)
\end{array}\right]=c .
$$

with

$$
\begin{aligned}
V= & {\left[\begin{array}{cccc}
I_{n_{1}} & 0 & 0 & 0 \\
0 & 0 & 0 & 0 \\
0 & 0 & I_{n_{2}} & 0 \\
0 & 0 & 0 & 0
\end{array}\right], } \\
N(\varepsilon)= & {\left[\begin{array}{cccc}
0 & 0 & 0 & 0 \\
F_{1} & -I_{n_{1}} & \varepsilon F_{2} & 0 \\
0 & 0 & 0 & 0 \\
F_{2}^{T} & 0 & F_{3} & -I_{n_{2}}
\end{array}\right], } \\
c= & {\left[\begin{array}{c}
x_{0} \\
0 \\
z_{0} \\
0
\end{array}\right] . }
\end{aligned}
$$

The Chang decoupling transformation [10] is defined by

$$
\left[\begin{array}{l}
x(t, \varepsilon) \\
p(t, \varepsilon) \\
z(t, \varepsilon) \\
r(t, \varepsilon)
\end{array}\right]=\mathbf{T}(\varepsilon)\left[\begin{array}{l}
x_{s}(t, \varepsilon) \\
p_{s}(t, \varepsilon) \\
z_{f}(t, \varepsilon) \\
r_{f}(t, \varepsilon)
\end{array}\right]
$$

to be

$$
\mathbf{T}(\varepsilon)=\left[\begin{array}{cc}
I_{2 n_{1}} & \varepsilon M(\varepsilon) \\
-L(\varepsilon) & I_{2 n_{2}}-\varepsilon L(\varepsilon) M(\varepsilon)
\end{array}\right]
$$

so

$$
\mathbf{T}^{-\mathbf{1}}(\varepsilon)=\left[\begin{array}{cc}
I_{2 n_{1}}-\varepsilon M(\varepsilon) L(\varepsilon) & -\varepsilon M(\varepsilon) \\
L(\varepsilon) & I_{2 n_{2}}
\end{array}\right]
$$

where the $2 n_{2} \times 2 n_{1}$ matrix $L(\varepsilon)$ satisfies

$$
\mathbf{A}_{\mathbf{4}} L(\varepsilon)-\mathbf{A}_{\mathbf{3}}-\varepsilon L(\varepsilon)\left(\mathbf{A}_{\mathbf{1}}-\mathbf{A}_{\mathbf{2}} L(\varepsilon)\right)=0
$$

and the $2 n_{1} \times 2 n_{2}$ matrix $M(\varepsilon)$ is obtained from

$$
\begin{aligned}
M(\varepsilon) \mathbf{A}_{\mathbf{4}}- & \mathbf{A}_{\mathbf{2}}+\varepsilon M(\varepsilon) L(\varepsilon) \mathbf{A}_{\mathbf{2}}- \\
& \varepsilon\left(\mathbf{A}_{\mathbf{1}}-\mathbf{A}_{\mathbf{2}} L(\varepsilon)\right) M(\varepsilon)=0 .
\end{aligned}
$$

The unique solution to (23) exists under Assumption 2 (that matrix $\mathbf{A}_{4}$ is invertible). This solution can be found numerically as the limit of a sequence of solutions of linear equations or by applying the eigenvector method [22]. The former process can be accomplished using either fixed-point iterations or a Newton method [3], [19]. The fixed-point algorithm for solving (23) is given by

$$
\begin{gathered}
L^{(i+1)}(\varepsilon)=\mathbf{A}_{\mathbf{4}}^{-\mathbf{1}}\left[\mathbf{A}_{\mathbf{3}}+\varepsilon L^{(i)}(\varepsilon)\left(\mathbf{A}_{\mathbf{1}}-\mathbf{A}_{\mathbf{2}} L^{(i)}(\varepsilon)\right)\right] \\
L^{(0)}(\varepsilon)=L^{(0)}(0)=\mathbf{A}_{\mathbf{4}}^{-\mathbf{1}} \mathbf{A}_{\mathbf{3}}, \\
i=0,1,2, \ldots
\end{gathered}
$$

It can be easily shown that together

$$
\begin{gathered}
L(\varepsilon)=L^{(0)}(0)+O(\varepsilon), \text { and } L^{(0)}(0)=O(1) \\
\Rightarrow \quad L(\varepsilon)=O(1) .
\end{gathered}
$$

Having obtained a solution for $L(\varepsilon)$, one can obtain a solution for $M(\varepsilon)$ using the following iterative scheme

$$
\begin{aligned}
& M^{(i+1)}(\varepsilon)= {\left[\mathbf{A}_{\mathbf{2}}-\varepsilon M^{(i)}(\varepsilon) L(\varepsilon) \mathbf{A}_{\mathbf{2}}+\right.} \\
&\left.\varepsilon\left(\mathbf{A}_{\mathbf{1}}-\mathbf{A}_{\mathbf{2}} L(\varepsilon)\right) M^{(i)}(\varepsilon)\right] \mathbf{A}_{\mathbf{4}}^{-\mathbf{1}}, \\
& M^{(0)}(\varepsilon)= M^{(0)}(0)=\mathbf{A}_{\mathbf{2}} \mathbf{A}_{\mathbf{4}}^{-1}, \\
& i=0,1,2, \ldots
\end{aligned}
$$

It is easy to establish that together

$$
\begin{gathered}
M(\varepsilon)=M^{(0)}(0)+O(\varepsilon), \text { and } M^{(0)}(0)=O(1) \\
\Rightarrow \quad M(\varepsilon)=O(1) .
\end{gathered}
$$

In the following, we will use the partitioned matrices $L(\varepsilon)$ and $M(\varepsilon)$ and the partitioned product $L(\varepsilon) M(\varepsilon)$. Accordingly, we define

$$
\begin{aligned}
& L^{2 n_{2} \times 2 n_{1}}(\varepsilon)=\left[\begin{array}{ll}
L_{1}^{n_{2} \times n_{1}}(\varepsilon) & L_{2}^{n_{2} \times n_{1}}(\varepsilon) \\
L_{3}^{n_{2} \times n_{1}}(\varepsilon) & L_{4}^{n_{2} \times n_{1}}(\varepsilon)
\end{array}\right], \\
& M^{2 n_{1} \times 2 n_{2}}(\varepsilon)=\left[\begin{array}{ll}
M_{1}^{n_{1} \times n_{2}}(\varepsilon) & M_{2}^{n_{1} \times n_{2}}(\varepsilon) \\
M_{3}^{n_{1} \times n_{2}}(\varepsilon) & M_{4}^{n_{1} \times n_{2}}(\varepsilon)
\end{array}\right]
\end{aligned}
$$


and

$$
\begin{aligned}
(L(\varepsilon) M(\varepsilon))^{2 n_{2} \times 2 n_{2}} & =H(\varepsilon) \\
& =\left[\begin{array}{lll}
H_{1}^{n_{2} \times n_{2}}(\varepsilon) & H_{2}^{n_{2} \times n_{2}}(\varepsilon) \\
H_{3}^{n_{2} \times n_{2}}(\varepsilon) & H_{4}^{n_{2} \times n_{2}}(\varepsilon)
\end{array}\right] .
\end{aligned}
$$

Applying the Chang transformation [10] to the two-point boundary value problem defined by (16)-(19) transforms the system into new coordinates where the slow and fast state variables are dynamically decoupled, that is,

$$
\left[\begin{array}{l}
\dot{x}_{s}(t, \varepsilon) \\
\dot{p}_{s}(t, \varepsilon)
\end{array}\right]=\left(\mathbf{A}_{\mathbf{1}}-\mathbf{A}_{\mathbf{2}} L(\varepsilon)\right)\left[\begin{array}{l}
x_{s}(t, \varepsilon) \\
p_{s}(t, \varepsilon)
\end{array}\right]
$$

and

$$
\varepsilon\left[\begin{array}{c}
\dot{z}_{f}(t, \varepsilon) \\
\dot{r}_{f}(t, \varepsilon)
\end{array}\right]=\left(\mathbf{A}_{\mathbf{4}}+\varepsilon L(\varepsilon) \mathbf{A}_{\mathbf{2}}\right)\left[\begin{array}{c}
z_{f}(t, \varepsilon) \\
r_{f}(t, \varepsilon)
\end{array}\right]
$$

with boundary conditions satisfying

$$
V_{1}(\varepsilon)\left[\begin{array}{c}
x_{s}(0, \varepsilon) \\
p_{s}(0, \varepsilon) \\
z_{f}(0, \varepsilon) \\
r_{f}(0, \varepsilon)
\end{array}\right]+N_{1}(\varepsilon)\left[\begin{array}{c}
x_{s}\left(t_{f}, \varepsilon\right) \\
p_{s}\left(t_{f}, \varepsilon\right) \\
z_{f}\left(t_{f}, \varepsilon\right) \\
r_{f}\left(t_{f}, \varepsilon\right)
\end{array}\right]=c
$$

with

$$
\begin{aligned}
V_{1}(\varepsilon) & =V \mathbf{T}(\varepsilon) \\
& =\left[\begin{array}{cccc}
I_{n_{1}} & 0 & \varepsilon M_{1}(\varepsilon) & \varepsilon M_{2}(\varepsilon) \\
0 & 0 & 0 & 0 \\
-L_{1}(\varepsilon) & -L_{2}(\varepsilon) & I_{n_{2}}-\varepsilon H_{1}(\varepsilon) & -\varepsilon H_{2}(\varepsilon) \\
0 & 0 & 0 & 0
\end{array}\right]
\end{aligned}
$$

and

$$
\begin{aligned}
N_{1}(\varepsilon) & =N(\varepsilon) \mathbf{T}(\varepsilon) \\
& =\left[\begin{array}{cccc}
0 & 0 & 0 & 0 \\
\alpha_{1}(\varepsilon) & \alpha_{2}(\varepsilon) & \alpha_{3}(\varepsilon) & \alpha_{4}(\varepsilon) \\
0 & 0 & 0 & 0 \\
\beta_{1}(\varepsilon) & \beta_{2}(\varepsilon) & \beta_{3}(\varepsilon) & \beta_{4}(\varepsilon)
\end{array}\right]
\end{aligned}
$$

where

$$
\begin{aligned}
& \alpha_{1}(\varepsilon)=F_{1}-\varepsilon F_{2} L_{1}(\varepsilon) \\
& \alpha_{2}(\varepsilon)=-I_{n_{1}}-\varepsilon F_{2} L_{2}(\varepsilon) \\
& \alpha_{3}(\varepsilon)=\varepsilon\left(F_{1} M_{1}(\varepsilon)-M_{3}(\varepsilon)+F_{2}\left(I-\varepsilon H_{1}(\varepsilon)\right)\right) \\
& \alpha_{4}(\varepsilon)=\varepsilon\left(F_{1} M_{2}(\varepsilon)-M_{4}(\varepsilon)-\varepsilon F_{2} H_{2}(\varepsilon)\right),
\end{aligned}
$$

and

$$
\begin{aligned}
& \beta_{1}(\varepsilon)=F_{2}^{T}-F_{3} L_{1}(\varepsilon)+L_{3}(\varepsilon) \\
& \beta_{2}(\varepsilon)=-F_{3} L_{2}(\varepsilon)+L_{4}(\varepsilon) \\
& \beta_{3}(\varepsilon)=F_{3}\left(I_{n_{2}}-\varepsilon H_{1}(\varepsilon)\right)+\varepsilon\left(F_{2}^{T} M_{1}(\varepsilon)+H_{3}(\varepsilon)\right) \\
& \beta_{4}(\varepsilon)=-I_{n_{2}}+\varepsilon\left(F_{2}^{T} M_{2}(\varepsilon)-F_{3} H_{2}(\varepsilon)+H_{4}(\varepsilon)\right) .
\end{aligned}
$$

Rewriting (30) and (31) to display the block structure, we have

$$
\left[\begin{array}{c}
\dot{x}_{s}(t, \varepsilon) \\
\dot{p}_{s}(t, \varepsilon)
\end{array}\right]=\left[\begin{array}{ll}
a_{1}(\varepsilon) & a_{2}(\varepsilon) \\
a_{3}(\varepsilon) & a_{4}(\varepsilon)
\end{array}\right]\left[\begin{array}{l}
x_{s}(t, \varepsilon) \\
p_{s}(t, \varepsilon)
\end{array}\right]
$$

$$
\varepsilon\left[\begin{array}{c}
\dot{z}_{f}(t, \varepsilon) \\
\dot{r}_{f}(t, \varepsilon)
\end{array}\right]=\left[\begin{array}{ll}
b_{1}(\varepsilon) & b_{2}(\varepsilon) \\
b_{3}(\varepsilon) & b_{4}(\varepsilon)
\end{array}\right]\left[\begin{array}{c}
z_{f}(t, \varepsilon) \\
r_{f}(t, \varepsilon)
\end{array}\right]
$$

where

$$
\begin{aligned}
& a_{1}(\varepsilon)=A_{1}-A_{2} L_{1}(\varepsilon)+S_{12} L_{3}(\varepsilon), \\
& a_{2}(\varepsilon)=-S_{1}-A_{2} L_{2}(\varepsilon)+S_{12} L_{4}(\varepsilon), \\
& a_{3}(\varepsilon)=-Q_{1}+Q_{2} L_{1}(\varepsilon)+A_{3}^{T} L_{3}(\varepsilon), \\
& a_{4}(\varepsilon)=-A_{1}^{T}+Q_{2} L_{2}(\varepsilon)+A_{3}^{T} L_{4}(\varepsilon),
\end{aligned}
$$

and

$$
\begin{aligned}
& b_{1}(\varepsilon)=A_{4}+\varepsilon\left(L_{1}(\varepsilon) A_{2}-L_{2}(\varepsilon) Q_{2}\right), \\
& b_{2}(\varepsilon)=-S_{2}-\varepsilon\left(L_{1}(\varepsilon) S_{12}+L_{2}(\varepsilon) A_{3}^{T}\right), \\
& b_{3}(\varepsilon)=-Q_{3}+\varepsilon\left(L_{3}(\varepsilon) A_{2}-L_{4}(\varepsilon) Q_{2}\right), \\
& b_{4}(\varepsilon)=-A_{4}^{T}-\varepsilon\left(L_{3}(\varepsilon) S_{12}+L_{4}(\varepsilon) A_{3}^{T}\right) .
\end{aligned}
$$

Solutions of the two-point boundary value problem (37)(40) with boundary conditions defined in (32)-(36) can be obtained as shown below. From the terminal conditions (32)(36) we have

$$
\begin{aligned}
\alpha_{2} p_{s}\left(t_{f}, \varepsilon\right)= & -\alpha_{1} x_{s}\left(t_{f}, \varepsilon\right)-\varepsilon \tilde{\alpha}_{3} z_{f}\left(t_{f}, \varepsilon\right)- \\
& \varepsilon \tilde{\alpha}_{4} r_{f}\left(t_{f}, \varepsilon\right), \\
\beta_{4} r_{f}\left(t_{f}, \varepsilon\right)= & -\beta_{3} z_{f}\left(t_{f}, \varepsilon\right)-\beta_{1} x_{s}\left(t_{f}, \varepsilon\right)- \\
& \beta_{2} p_{s}\left(t_{f}, \varepsilon\right)
\end{aligned}
$$

where we have defined the new variables $\tilde{\alpha}_{3}:=\frac{1}{\varepsilon} \alpha_{3}$ and $\tilde{\alpha}_{4}:=\frac{1}{\varepsilon} \alpha_{4}$. Note that the matrices $\alpha_{2}(\varepsilon)$ and $\beta_{4}(\varepsilon)$ are nonsingular for sufficiently small values of $\varepsilon$ because they are $O(\varepsilon)$ perturbations of the identity matrix. These terminal conditions suggest that we look for the following relationships

$$
\begin{aligned}
p_{s}(t, \varepsilon)= & P_{s}(t, \varepsilon) x_{s}(t, \varepsilon)+\varepsilon g_{1}(t, \varepsilon) z_{f}(t, \varepsilon)+ \\
& \varepsilon g_{2}(t, \varepsilon) r_{f}(t, \varepsilon) \\
r_{f}(t, \varepsilon)= & P_{f}(t, \varepsilon) z_{f}(t, \varepsilon)+g_{3}(t, \varepsilon) x_{s}(t, \varepsilon)+ \\
& g_{4}(t, \varepsilon) p_{s}(t, \varepsilon)
\end{aligned}
$$

where, to satisfy (41) we need the following boundary conditions

$$
\begin{aligned}
& P_{s}\left(t_{f}, \varepsilon\right)=-\alpha_{2}^{-1}(\varepsilon) \alpha_{1}(\varepsilon), \\
& g_{1}\left(t_{f}, \varepsilon\right)=-\alpha_{2}^{-1}(\varepsilon) \tilde{\alpha}_{3}(\varepsilon), \\
& g_{2}\left(t_{f}, \varepsilon\right)=-\alpha_{2}^{-1}(\varepsilon) \tilde{\alpha}_{4}(\varepsilon), \\
& P_{f}\left(t_{f}, \varepsilon\right)=-\beta_{4}^{-1}(\varepsilon) \beta_{3}(\varepsilon), \\
& g_{3}\left(t_{f}, \varepsilon\right)=-\beta_{4}^{-1}(\varepsilon) \beta_{1}(\varepsilon), \\
& g_{4}\left(t_{f}, \varepsilon\right)=-\beta_{4}^{-1}(\varepsilon) \beta_{2}(\varepsilon) .
\end{aligned}
$$

Taking the derivatives of (42) and using (37)-(38) we obtain two nonsymmetric differential Riccati equations corresponding to the slow and fast variables, respectively,

$$
\begin{aligned}
-\dot{P}_{s}(t, \varepsilon)= & P_{s}(t, \varepsilon) a_{1}(\varepsilon)-a_{4}(\varepsilon) P_{s}(t, \varepsilon)-a_{3} \\
& (\varepsilon)+P_{s}(t, \varepsilon) a_{2}(\varepsilon) P_{s}(t, \varepsilon) \\
& P_{s}\left(t_{f}, \varepsilon\right)=-\alpha_{2}^{-1}(\varepsilon) \alpha_{1}(\varepsilon) \\
-\varepsilon \dot{P}_{f}(t, \varepsilon)= & P_{f}(t, \varepsilon) b_{1}(\varepsilon)-b_{4}(\varepsilon) P_{f}(t, \varepsilon)-b_{3} \\
& (\varepsilon)+P_{f}(t, \varepsilon) b_{2}(\varepsilon) P_{f}(t, \varepsilon) \\
& P_{f}\left(t_{f}, \varepsilon\right)=-\beta_{4}^{-1}(\varepsilon) \beta_{3}(\varepsilon)
\end{aligned}
$$


and two linear initial value problems

$$
\begin{aligned}
\varepsilon \dot{g}_{1}(t, \varepsilon)= & -g_{1}(t, \varepsilon) b_{1}(\varepsilon) \\
& +\varepsilon\left[a_{4}(\varepsilon)-P_{s}(t, \varepsilon) a_{2}(\varepsilon)\right] g_{1}(t, \varepsilon) \\
& -g_{2}(t, \varepsilon) b_{3}(\varepsilon) \\
& g_{1}\left(t_{f}, \varepsilon\right)=-\alpha_{2}^{-1}(\varepsilon) \tilde{\alpha}_{3}(\varepsilon) \\
\varepsilon \dot{g}_{2}(t, \varepsilon)= & -g_{1}(t, \varepsilon) b_{2}(\varepsilon)-g_{2}(t, \varepsilon) b_{4}(\varepsilon) \\
& +\varepsilon\left[a_{4}(\varepsilon)-P_{s}(t, \varepsilon) a_{2}(\varepsilon)\right] g_{2}(t, \varepsilon) \\
& g_{2}\left(t_{f}, \varepsilon\right)=-\alpha_{2}^{-1}(\varepsilon) \tilde{\alpha}_{4}(\varepsilon)
\end{aligned}
$$

and

$$
\begin{aligned}
\varepsilon \dot{g}_{3}(t, \varepsilon)= & {\left[b_{4}(\varepsilon)-P_{f}(t, \varepsilon) b_{2}(\varepsilon)\right] g_{3}(t, \varepsilon)-} \\
& \varepsilon g_{3}(t, \varepsilon) a_{1}(\varepsilon)-\varepsilon g_{4}(t, \varepsilon) a_{3}(\varepsilon), \\
& g_{3}\left(t_{f}, \varepsilon\right)=-\beta_{4}^{-1}(\varepsilon) \beta_{1}(\varepsilon) \\
\varepsilon \dot{g}_{4}(t, \varepsilon)= & {\left[b_{4}(\varepsilon)-P_{f}(t, \varepsilon) b_{2}(\varepsilon)\right] g_{4}(t, \varepsilon)-} \\
& \varepsilon g_{3}(t, \varepsilon) a_{2}(\varepsilon)-\varepsilon g_{4}(t, \varepsilon) a_{4}(\varepsilon), \\
& g_{4}\left(t_{f}, \varepsilon\right)=-\beta_{4}^{-1}(\varepsilon) \beta_{2}(\varepsilon) .
\end{aligned}
$$

These differential equations in $t$ and $\varepsilon$ satisfy the following conditions at $t=t_{f}$ and $\varepsilon=0$ :

$$
\begin{aligned}
& g_{1}\left(t_{f}, 0\right)=-F_{1} M_{1}+M_{3}-F_{2}, \\
& g_{2}\left(t_{f}, 0\right)=-F_{1} M_{2}+M_{4} \\
& g_{3}\left(t_{f}, 0\right)=F_{2}^{T}-F_{3} L_{1}+L_{3}, \\
& g_{4}\left(t_{f}, 0\right)=F_{3} L_{2}+L_{4} .
\end{aligned}
$$

Fridman [23] used the integral manifold approach of Sobolev [24] to derive pure-slow and pure-fast, reducedorder, differential matrix Riccati equations similar to (44)(45) with different terminal conditions. However, the transformation of Sobolev is a function of $P_{f}(t, \varepsilon)$, hence timevarying and not convenient for relating the original and new coordinates. In the following, we will show that the transformation introduced in this paper is time invariant (see (51)) so that we can easily establish the relation between the new and original coordinates and derive a formula that relates the solution of the original full order singularly perturbed differential Riccati equation and the solutions of the pure-slow and pure-fast reduced-order differential Riccati equations.

The relationship between the original coordinates, $x(t, \varepsilon), z(t, \varepsilon), p(t, \varepsilon)$ and $q(t, \varepsilon)$, and the new coordinates $x_{s}(t, \varepsilon), z_{f}(t, \varepsilon), p_{s}(t, \varepsilon)$ and $r_{f}(t, \varepsilon)$, is given by a time invariant similarity transformation $\Pi(\varepsilon)$ where

$$
\begin{aligned}
{\left[\begin{array}{l}
x_{s}(t, \varepsilon) \\
z_{f}(t, \varepsilon) \\
p_{s}(t, \varepsilon) \\
r_{f}(t, \varepsilon)
\end{array}\right] } & =\underbrace{\left[\begin{array}{cccc}
I_{n_{1}} & 0 & 0 & 0 \\
0 & 0 & I_{n_{2}} & 0 \\
0 & I_{n_{1}} & 0 & 0 \\
0 & 0 & 0 & I_{n_{2}}
\end{array}\right]}_{E_{1}}\left[\begin{array}{l}
x_{s}(t, \varepsilon) \\
p_{s}(t, \varepsilon) \\
z_{f}(t, \varepsilon) \\
r_{f}(t, \varepsilon)
\end{array}\right] \\
& =E_{1} \mathbf{T}^{-1}(\varepsilon)\left[\begin{array}{l}
x(t, \varepsilon) \\
p(t, \varepsilon) \\
z(t, \varepsilon) \\
r(t, \varepsilon)
\end{array}\right]
\end{aligned}
$$

$$
\begin{aligned}
& =E_{1} \mathbf{T}^{-1}(\varepsilon) E_{2}\left[\begin{array}{l}
x(t, \varepsilon) \\
z(t, \varepsilon) \\
p(t, \varepsilon) \\
q(t, \varepsilon)
\end{array}\right] \\
& =\Pi(\varepsilon)\left[\begin{array}{l}
x(t, \varepsilon) \\
z(t, \varepsilon) \\
p(t, \varepsilon) \\
q(t, \varepsilon)
\end{array}\right]
\end{aligned}
$$

where

$$
E_{2}=\left[\begin{array}{cccc}
I_{n_{1}} & 0 & 0 & 0 \\
0 & 0 & I_{n_{1}} & 0 \\
0 & I_{n_{2}} & 0 & 0 \\
0 & 0 & 0 & \frac{1}{\varepsilon} I_{n_{2}}
\end{array}\right],
$$

and $E_{1}(\varepsilon)$ is defined as indicated, $\mathbf{T}^{-1}(\varepsilon)$ is defined in (20) and $\Pi(\varepsilon)=E_{1}(\varepsilon) T^{-1}(\varepsilon) E_{2}(\varepsilon)$.

Since $\Pi(\varepsilon)$ is an invertible matrix we also have the inverse coordinate transform $\Omega(\varepsilon)$ given by

$$
\begin{aligned}
{\left[\begin{array}{l}
x(t, \varepsilon) \\
z(t, \varepsilon) \\
p(t, \varepsilon) \\
q(t, \varepsilon)
\end{array}\right] } & =\Pi^{-1}(\varepsilon)\left[\begin{array}{l}
x_{s}(t, \varepsilon) \\
z_{f}(t, \varepsilon) \\
p_{s}(t, \varepsilon) \\
r_{f}(t, \varepsilon)
\end{array}\right] \\
& =\Omega(\varepsilon)\left[\begin{array}{l}
x_{s}(t, \varepsilon) \\
z_{f}(t, \varepsilon) \\
p_{s}(t, \varepsilon) \\
r_{f}(t, \varepsilon)
\end{array}\right] \\
& =\left[\begin{array}{ll}
\Omega_{1}(\varepsilon) & \Omega_{2}(\varepsilon) \\
\Omega_{3}(\varepsilon) & \Omega_{4}(\varepsilon)
\end{array}\right]\left[\begin{array}{l}
x_{s}(t, \varepsilon) \\
z_{f}(t, \varepsilon) \\
p_{s}(t, \varepsilon) \\
r_{f}(t, \varepsilon)
\end{array}\right] .
\end{aligned}
$$

From (52), we have

$$
\left[\begin{array}{l}
x(t, \varepsilon) \\
z(t, \varepsilon)
\end{array}\right]=\Omega_{1}(\varepsilon)\left[\begin{array}{l}
x_{s}(t, \varepsilon) \\
z_{f}(t, \varepsilon)
\end{array}\right]+\Omega_{2}(\varepsilon)\left[\begin{array}{l}
p_{s}(t, \varepsilon) \\
r_{f}(t, \varepsilon)
\end{array}\right]
$$

and

$$
\left[\begin{array}{l}
p(t, \varepsilon) \\
q(t, \varepsilon)
\end{array}\right]=\Omega_{3}(\varepsilon)\left[\begin{array}{l}
x_{s}(t, \varepsilon) \\
z_{f}(t, \varepsilon)
\end{array}\right]+\Omega_{4}(\varepsilon)\left[\begin{array}{l}
p_{s}(t, \varepsilon) \\
r_{f}(t, \varepsilon)
\end{array}\right] .
$$

The state and costate equations of the original system (14)(15) are related through the solution of the original differential Riccati equation (6), that is

$$
\left[\begin{array}{l}
p(t, \varepsilon) \\
q(t, \varepsilon)
\end{array}\right]=P(t, \varepsilon)\left[\begin{array}{l}
x(t, \varepsilon) \\
z(t, \varepsilon)
\end{array}\right]
$$

We can rewrite (42) in matrix form as follows:

$$
\begin{gathered}
{\left[\begin{array}{cc}
I_{n_{1}} & -\varepsilon g_{2}(t, \varepsilon) \\
-g_{4}(t, \varepsilon) & I_{n_{2}}
\end{array}\right]\left[\begin{array}{l}
p_{s}(t, \varepsilon) \\
r_{f}(t, \varepsilon)
\end{array}\right]=} \\
{\left[\begin{array}{cc}
P_{s}(t, \varepsilon) & \varepsilon g_{1}(t, \varepsilon) \\
g_{3}(t, \varepsilon) & P_{f}(t, \varepsilon)
\end{array}\right]\left[\begin{array}{l}
x_{s}(t, \varepsilon) \\
z_{f}(t, \varepsilon)
\end{array}\right] .}
\end{gathered}
$$

Then from (53) through (56) we can derive the formula that relates the solution of the original differential Riccati equation (6) and the solutions of the pure-slow and pure-fast differential Riccati equations (44) through (45) as

$$
\begin{aligned}
P(t, \varepsilon)= & \left(\Omega_{3}(\varepsilon)+\Omega_{4}(\varepsilon) \Psi(t, \varepsilon) \Theta(t, \varepsilon)\right) \\
& \times\left(\Omega_{1}(\varepsilon)+\Omega_{2}(\varepsilon) \Psi(t, \varepsilon) \Theta(t, \varepsilon)\right)^{-1}
\end{aligned}
$$


where

$$
\Psi(t, \varepsilon)=\left[\begin{array}{cc}
I_{n_{1}} & -\varepsilon g_{2}(t, \varepsilon) \\
-g_{4}(t, \varepsilon) & I_{n_{2}}
\end{array}\right]^{-1}
$$

and

$$
\Theta(t, \varepsilon)=\left[\begin{array}{cc}
P_{s}(t, \varepsilon) & \varepsilon g_{1}(t, \varepsilon) \\
g_{3}(t, \varepsilon) & P_{f}(t, \varepsilon)
\end{array}\right]
$$

The complete proof of this result is lengthy and is omitted due to space constraints. In the proof we establish the existence of all matrix inverses in (57) and the existence, uniqueness and boundedness of $P_{f}$ and $P_{s}$. We then show $\mathrm{O}(1)$ boundedness of $g_{i}$ on compact sets. Finally we show that the existence and boundedness of the $g_{i}, P_{f}$, and $P_{s}$ on compact sets suffices to guarantee the existence of the required inverses.

\section{EXAMPLE: FLUID CATALYTIC REACTOR}

The fluid catalytic cracker[25] provides a fifth order practical example of a singularly perturbed system. The system has three fast variables and two slow variables.

The partitioned $A$ matrix is given by

$A=\left[\begin{array}{rr|rrr}-16.11 & -0.39 & 27.20 & 0 & 0 \\ 0.01 & -16.99 & 0 & 0 & 12.47 \\ \hline 15.11 & 0 & -53.60 & -16.57 & 71.78 \\ -53.36 & 0 & 0 & -107.20 & 232.11 \\ 2.27 & 69.10 & 0 & 2.273 & -102.99\end{array}\right]$

which has eigenvalues $-129.0847,-82.8581,-74.3201$, -2.8488 , and -7.7782 . The ratio of the fastest "slow" eigenvalue to slowest "fast" eigenvalue is 0.1047 , so this system is a good candidate for use of the mathematical technique described in this paper.

The partitioned $B$ matrix is

$$
B=\left[\begin{array}{rr}
11.12 & -12.60 \\
-3.61 & 3.36 \\
\hline-21.91 & 0 \\
-53.60 & 0 \\
69.10 & 0
\end{array}\right]
$$

In the computations, done in Matlab, we used $\varepsilon=0.1 \mathrm{We}$ used cost weighting matrices $Q=I$, and $R=I$. For visual clarity we have plotted only the diagonal elements of these matrices.

The evolution of the solution matrices $P_{f}$ and $P_{s}$ of the fast and slow subsystem differential Riccati equations is illustrated in Figures 1 and 2. The resulting solution to the original differential Riccati equation constructed using (57) from the solutions of the fast and slow subsystems is shown in Figure 3. The error is shown in Figure 4.

The figures presented here show excellent agreement between the $P(t, \varepsilon)$ computed by combining the fast and slow matrices $P_{f}(t, \varepsilon)$ and $P_{s}(t, \varepsilon)$, respectively.

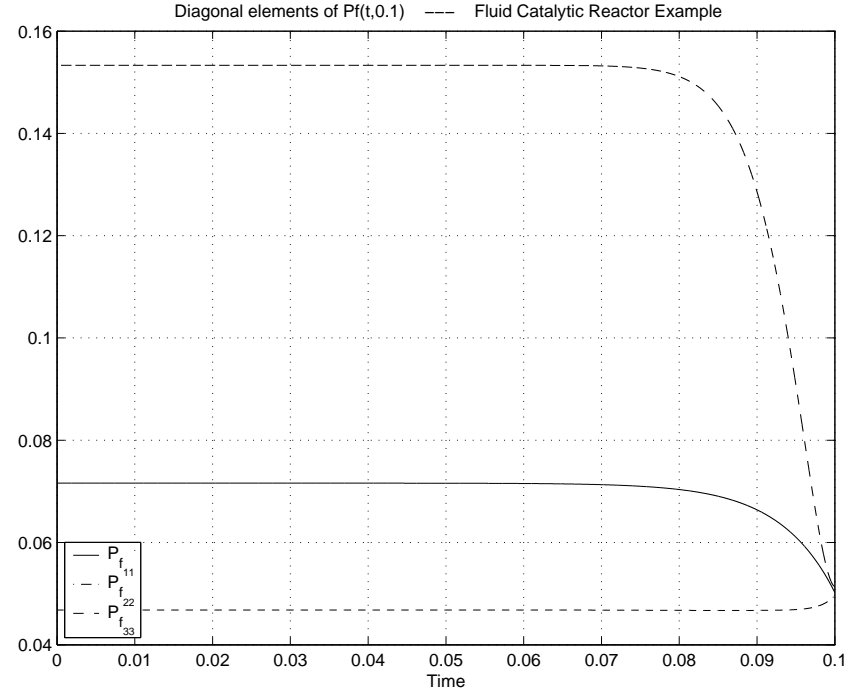

Fig. 1. Solution $P_{f}$ of the fast subsystem differential Riccati equation, for the catalytic cracker example.

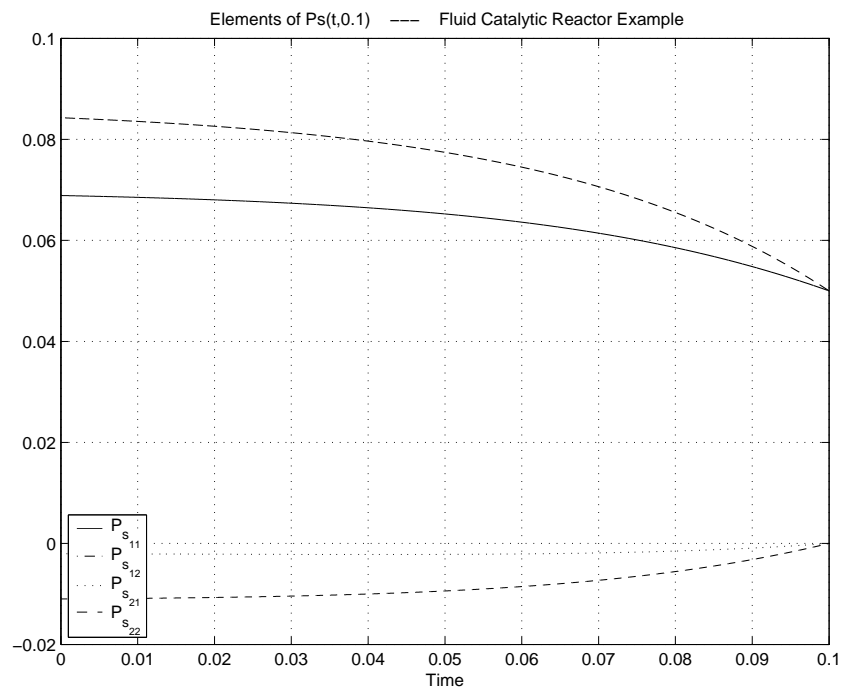

Fig. 2. Solution $P_{s}$ of the slow subsystem differential Riccati equation for the catalytic cracker example.

\section{CONCLUSIONS}

Reduced-order pure-slow and pure-fast matrix differential Riccati equations of singularly perturbed systems were derived by decoupling the singularly perturbed differential matrix Riccati equation into the regular differential matrix Riccati equation pure-slow and the stiff differential matrix Riccati equation pure-fast. The solution of the original singularly perturbed matrix Riccati differential equation is then given in terms of solutions of the pure-slow and purefast reduced-order differential matrix Riccati equations and solutions of two reduced-order initial value problems. The significance of this result is that it allows the solution to be obtained while solving only a differential matrix Riccati equation of dimension $n_{1}$ and one of dimension $n_{2}$ rather than the original equation of dimension $n_{1}+n_{2}$. Potential 


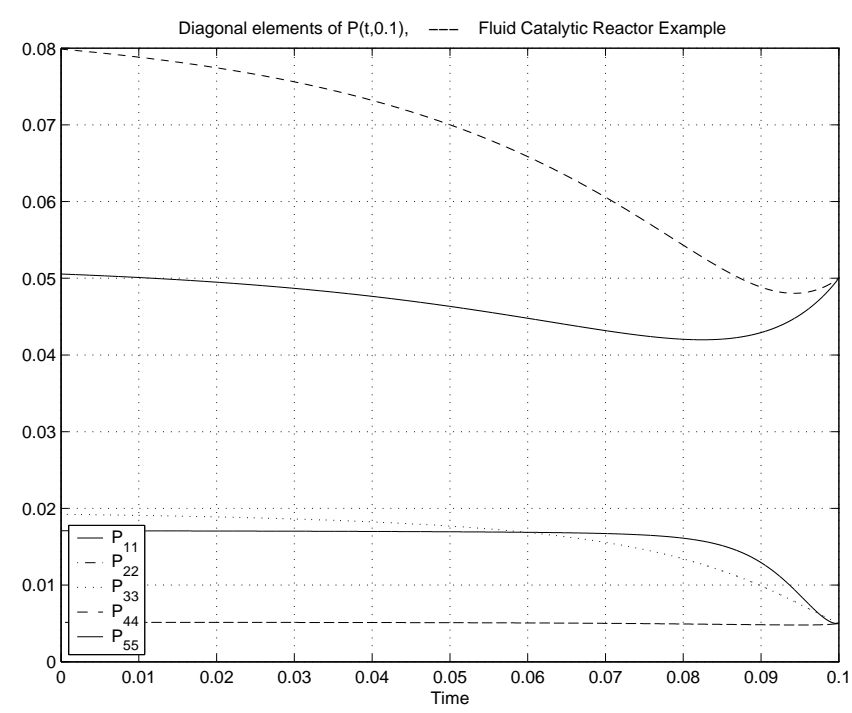

Fig. 3. Solution of the original differential Riccati equation generated from $P_{f}$ and $P_{s}$.

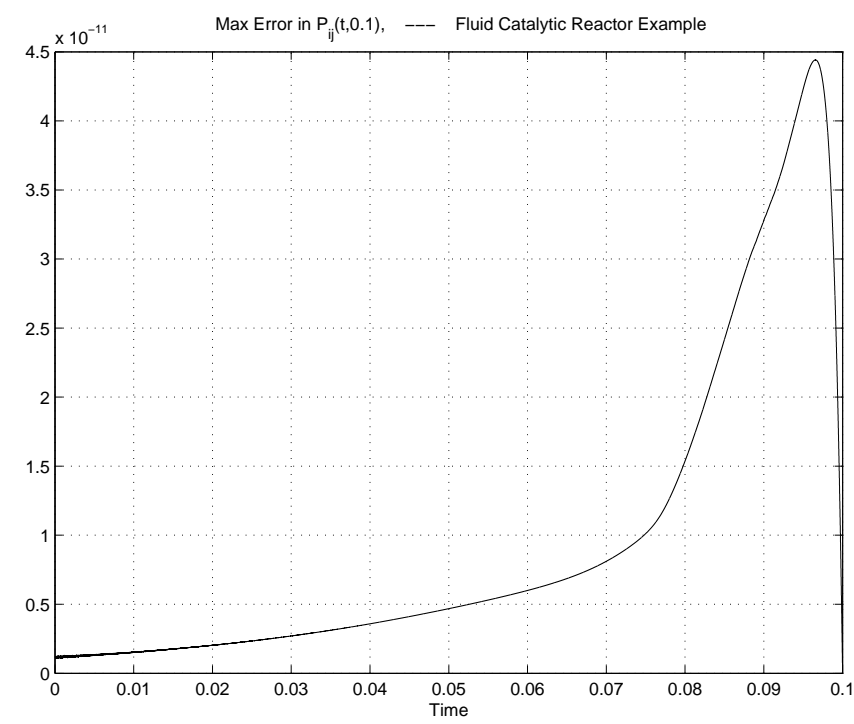

Fig. 4. Maximum error over elements of $P_{i j}$ as a function of time.

applications include optimal filtering and optimal control of singularly perturbed linear systems.

\section{REFERENCES}

[1] R. O'Malley, Introduction to Singular Perturbations. New York: Academic Press, 1974.

[2] R. O'Malley, Singular Perturbations for Ordinary Differential Equations. New York: Springer-Verlag, 1991.

[3] P. Kokotovic, H. Khalil, and J. O'Reilly, Singular Perturbation Methods in Control: Analysis and Design. Orlando: Academic Press, 1986.

[4] P. V. Kokotovic and H. K. Khalil, Singular Perturbations in Systems and Controls. New York: IEEE Press, 1986.

[5] Z. Gajic and X. Shen, Parallel Algorithms for Optimal Control of Large Scale Linear Systems. London: Springer Verlag, 1993.

[6] Z. Gajic and M. Lim, Optimal Control of Linear Singularly Perturbed Systems and Applications: High Accuracy Techniques. New York: Marcel Dekker, 2001

[7] B. Kreiss and H. Kreiss, "Numerical methods for singular perturbation problems," SIAM J. Numer. Anal., vol. 18, pp. 262-276, 1981.

[8] A. Tikhonov, "On the dependence of the solutions of differential equations on a small parameter," Mat. Sb., vol. 22, pp. 193-204, 1948.

[9] A. Vasileva and V. Butuzov, Asymptotic Expansions of Solutions of Singularly Perturbed Differential Equations. Moscow: Nauka, 1973.

[10] K. Chang, "Singular perturbations of a general boundary value problem," SIAM J. Math. Anal., vol. 3, pp. 520-526, 1972.

[11] M. Qureshi and Z. Gajic, "A new version of the Chang transformation," IEEE Trans. Automatic Control, vol. 37, pp. 800-801, 1992.

[12] D. Smith, "Decoupling and order reduction via the Riccati transformation," SIAM Review, vol. 29, pp. 91-113, 1987.

[13] T. H. Hsieh and Z. Gajic, "An algorithm for solving the singularly perturbed H-infinity algebraic Riccati equation," Computers \& Mathematics With Applications, vol. 36, pp. 69-77, Sept. 1998.

[14] E. Fridman, "Exact slow-fast decomposition of a class of non-linear singularly perturbed optimal control problems via invariant manifolds,' International Journal of Control, vol. 72, pp. 1609-1618, Nov. 1999.

[15] E. Fridman, "Exact slow-fast decomposition of the nonlinear singularly perturbed optimal control problem," Systems \& Control Letters, vol. 40, pp. 121-131, June 2000.

[16] P. Kokotovic and R. Yackel, "Singular perturbation of linear regulators: basic theorems," IEEE Trans. Automatic Control, vol. 17, pp. 29-37, 1972.

[17] H. Khalil, "Feedback control of nonstandard singularly perturbed systems," IEEE Transactions on Automatic Control, vol. 34, pp. 10521060, 1989.

[18] R. Wilde and P. Kokotovic, "A dichotomy in linear control theory," IEEE Transactions on Automatic Control, vol. 17, pp. 382-383, 1972.

[19] T. Grodt and Z. Gajic, "The recursive reduced order numerical solution of the singularly perturbed matrix differential Riccati equation," IEEE Trans. Automatic Control, vol. 33, pp. 751-754, 1988.

[20] R. Yackel and P. Kokotovic, "A boundary layer method for the matrix Riccati equation," IEEE Trans. Automatic Control, vol. 18, pp. 17-24, 1973.

[21] W. Su, Z. Gajic, and X. Shen, "The exact slow-fast decomposition of the algebraic Riccati equation of singularly perturbed systems," IEEE Trans. Automatic Control, vol. 37, pp. 1456-1459, 1992.

[22] V. Kecman, S. Bingulac, and Z. Gajic, "Eigenvector approach for order reduction of singularly perturbed linear-quadratic optimal control problems," Automatica, vol. 35, pp. 151-158, 1999.

[23] E. Fridman, "Near-optimal $H^{\infty}$ control of linear singularly perturbed systems," IEEE Trans. on Automatic Control, vol. 41, pp. 236-240, 1996.

[24] V. Sobolev, "Integral manifolds and decomposition of singularly perturbed systems," Systems \& Control Letters, vol. 5, pp. 169-179, 1984.

[25] Y. Arkun and S. Ramakrishnan, "Bounds on the optimum quadratic cost of structure-constrained controllers," IEEE Transactions on Automatic Control, vol. 28, pp. 924-927, Sept. 1983. 